INVESTIGACIÓN / RESEARCH

Recibido: 24/04/2015 Aceptado: 19/06/2015 Publicado: 15/09/2015

\title{
VALORACIONES A LA COMUNICACIÓN CIENTÍFICA: REVISTA CUBANA DE GEOGRAFÍA
}

Manuel Paulino Linares Herrera ${ }^{1}$ : Academia de Ciencias, La Habana, Cuba mplinares106@gmail.com

Ernesto Budiño Rodríguez: Instituto de Geografía Tropical, La Habana, Cuba ernesto@geotech.cu

Orestes Sardiñas Gómez: Instituto de Geografía Tropical, La Habana, Cuba oreste@geotech.cu

\section{RESUMEN:}

La incursión de nuevas tecnologías de la información y la comunicación han traído al campo de la comunicación científica nuevos paradigmas para su desarrollo. Se revisa a través de un balance estadístico, el curso de las revistas temáticas en ciencias geográficas, en Portales de difusión científica, como; Redalyc y Latindex. Además se refiere la indagación, sobre la tipología de los documentos científicos y sus tendencias actuales. Se concluye sobre las estrategias qué se llevan a cabo para potenciar a la Revista Cubana de Geografía en los nuevos contextos, que van desde su acreditación con la certificación del Ministerio de Ciencia, Tecnología y medioambiente (CITMA) hasta el estudio de la implementación del gestor editorial Open Jiournal System (OJS).

PALABRAS CLAVE: revistas científicas - revista cubana de geografía - producción científica - Cuba - comunicación de resultados

\section{REVIEWS A SCIENTIFIC COMMUNICATION: CUBAN JOURNAL OF GEOGRAPHY}

\section{ABSTRACT:}

\footnotetext{
1 Manuel Paulino Linares Herrera: profesor en la facultad de Comunicación, especialista e investigador y Editor de publicaciones Científicas: Bibliotecas. Anales de la Investigación y otras fue editor de la Revista Cubana de Geografía (edición 2013)

Correo: mplinares106@gmail.com
} 
The incursion of new information technologies and communication have brought to the field of scientific communication paradigms for development. It is reviewed by a statistical review, the course of thematic magazines in geographical sciences, scientific dissemination portals such as; Redalyc and Latindex. Also referred the inquiry, on the typology of scientific documents and current trends. It is concluded on the strategies that are held to empower the Cuban Journal of Geography in new contexts, ranging from accreditation to certification by the Ministry of Science, Technology and Environment (CITMA) to study the deployment manager Open Jiournal Editorial System (OJS).

KEYWORDS: scientific journals - Cuban geography magazine - scientific production - Cuba - communication of results

\section{INTRODUCCIÓN}

El desarrollo de los países depende de su capacidad para generar y utilizar conocimientos. Es un principio paradigmático en la sociedad de la información. Es determinante y medible desde los análisis estadísticos nacionales y organizacionales. El conocimiento gestionado en función de la producción científica es concluyente para el status socio-económico de los países; potenciarlo y viabilizarlo es un deber social de todo investigador, especialista o profesional.

Para posibilitar la difusión de los conocimientos científicos, existen varias vías comunicativas, una de ellas son las revisitas científicas. En Cuba hay acreditadas unas 870 publicaciones periódicas que desarrollan contenidos informativos, especializados y científicos, de ellos, unas 350 son revistas científicas; todas estas publicaciones están inscritas en el Registro Nacional de Publicaciones Seriadas (RNPS) y responden a las disímiles temáticas de los saberes y las ciencias. En temas de revistas científicas, el organismo superior que certifica el cumplimiento de la comunicación hacia sus públicos con la calidad requerida, es el Ministerio de Ciencia, Tecnología y Medioambiente de Cuba (CITMA) a través de la resolución 59/2003.

Las revistas científicas validan el nuevo conocimiento, lo hacen público, lo convierten en patrimonio de la humanidad a través de los recursos y herramientas que ofrecen las tecnologías de la Información y la Comunicación (TIC), como es el caso del Medio de los Medios, la Internet, y toda su red de repositorios y bases de datos. La publicación de resultados es un determinante e importante proceso de gran impacto social.

\subsection{Cuba y su posicionamiento}

Cuba presenta situaciones atípica con relación al resto de los países latinoamericanos, que le obliga a implementar solidas estrategias en pos de insertarse en los ámbitos científicos; es sometida a un férreo bloqueo que atenta contra su desarrollo científico-social, pero a su vez, es realmente sorprendente su posición regional si tenemos en cuenta: primero, su población total y después la 
cantidad de científicos, estos, por miles de habitantes. Ambos parámetros son reconocidos a nivel internacional, pues derivan en significativos resultados en la gestión de la comunicación científica (publicaciones, posicionamiento, visibilidad e interacciones gestoras que derivan en proyectos, inversiones y capital humano altamente especializado).

El posicionamiento en difusión, es un indicador importante para la producción científica, uno de sus exponentes más emblemático y propiciador de altos índices de producción científica. En la comunidad científica Latinoamericana la Red de Revistas Científicas de América Latina y el Caribe, España y Portugal,(Redalyc) ubica a Cuba en el 8vo. lugar, según datos de 2012, evidenciados a partir del total de publicaciones cubanas que existen en este repositorio, 22 revistas en total . Los criterios que establece esta plataforma difusora están implementados en las publicaciones cubanas en correspondencia a los modelos de comunicación científica establecidos por la comunidad internacional. (ver figura 1.)

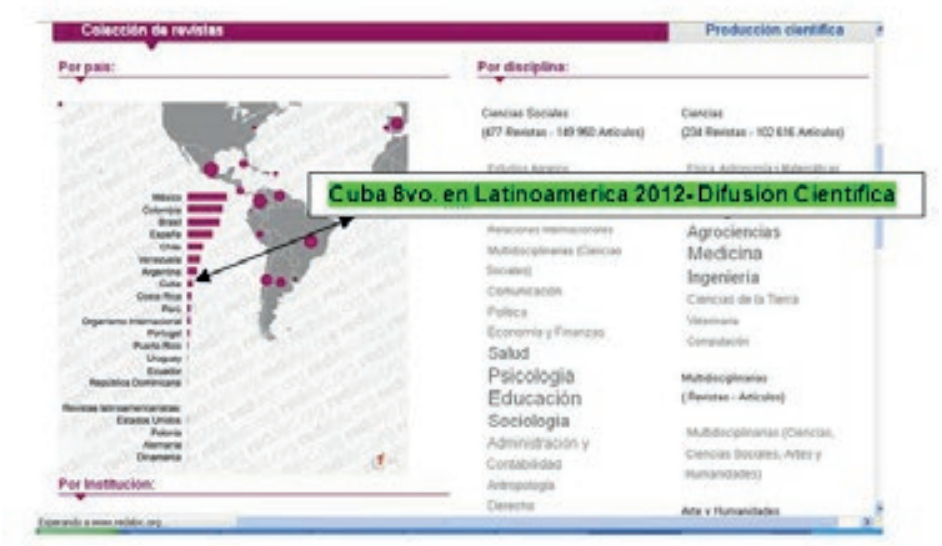

Figura 1. Fuente: http://redalyc.org/colecionderevistas/produccioncientifica/ Consultado el 24 de mayo de 2013

\section{OBJETIVO}

La investigación destaca como objetivo, la importancia de publicar los resultados de investigación, con vista a potenciar la producción científica y su impacto social. Para cumplimentar el objetivo se partió de un análisis documental y bibliográfico a través de la Web, para valorar el estado de la producción científica en las ciencias geográficas, el marco de estudio.

\section{METODOLOGÍA}

\subsection{Desde un análisis métrico}

El análisis documental y bibliográfico realizado ha derivado en interrogativas (cómo, cuándo) que obligan a trazar estrategias en pos de incrementar el desarrollo de la producción científica en las ciencias geográficas, el marco disciplinario de estudio. 
Un indicador de la producción científica evaluable en las políticas científicas y por organismo internacionales lo es el índice $\boldsymbol{H}$, aplicable en todas las ramas de las ciencias, no presenta este la misma dinámica en todas pues depende de varios factores, entre ellos la velocidad de la obsolescencia de las publicaciones científicas, que no es igual en todas las disciplinas, así como tampoco los patrones de citación. Se define el índice $\mathrm{H}$ como el balance entre el número de publicaciones y sus citas, para medir eficazmente el consumo e impacto de las investigaciones. (Dorta Contreras AJ, Álvarez Díaz LI, 2007)

\section{DISCUSIÓN}

\subsection{Plataformas de difusión científica}

En Cuba la dirección de Ciencias del CITMA, se encarga mediante los grupos de estudios cienciométricos, de estudiar, establecer políticas y comunicar sobre sus perspectivas. Estudios métricos realizados por los investigadores Dorta Contreras AJ, Álvarez Díaz LI, (2007) a la base de datos Scopus, asociada a la editorial internacional de información científica Thomson Reuters, concluyó con resultados alentadores, en dos de los investigadores cubanos estudiados; de un total de 320 documentos científicos realizados por cubanos en temas de salud y publicados en un período de 10 años, resultaron sus citas ser 4164, ocupando un lugar destacado en el ámbito iberoamericano, con el puesto 38 por autoría, en esta base de datos, reportada como de las primeras en el ranking mundial en temas de publicaciones científicas.

Como destacamos, la plataforma de difusión científica de acceso abierto Redalyc es un repositorio de información científica que gestiona la Universidad del Estado de México (UEA) y aloja en su sistema informativo un total de 809 revistas científicas, sumando todos sus contenidos son 280860 artículos, los cuales se agrupan en revistas de 157 países, iberoamericanas en su mayorías y otras estudiosas de temas latinoamericanos de otras latitudes, según reportan las estadísticas de 2012.

Existen en esta base 8390 artículos en las 19 revistas que tratan temas de Ciencias de la Tierra, y de Geografía Social (según las clasificaciones disciplinarias que exponen) coexisten 7 revistas de esta última temática totalizando 2435 artículos; de Estudios Ambientales hay 5 revistas con un total de 1423 artículos. No existiendo hasta el momento publicaciones científicas cubanas en estas temáticas registradas en el sistema de Información Científica (Redalyc). Las 22 revistas científicas cubanas registradas corresponden a otras disciplinas científicas, las cuales totalizan 8988 artículos, avalados por los criterios de evaluación del sistema, que se identifica con 12 aspectos de obligatorio cumplimiento, los cuales corresponden a la estructura y tratamiento de sus contenidos, (ver figura 1.)

Los criterios evaluativos se ajustan a las concepciones más recientes sobre comunicación y difusión científica. Desde los contenidos se ventilan tendencias de multipropósitos investigativos y con valor agregado, los cuales se manifiestan desde concepciones interdisciplinar, multidisciplinar y transdisciplinar en las indagaciones 
que se llevan a cabo, tendencia generalizada en todas las ciencias, subrayando, que es importante partir desde las conceptualizaciones disciplinarias, para interrelacionar los estudios y sus impactos.

Latindex, otra de las plataformas de gestión en difusión científica, actúa producto de la cooperación de una red de instituciones que funcionan de manera coordinada para reunir y diseminar información bibliográfica sobre las publicaciones científicas seriadas producidas en la región (Latinoamérica). Existen en su espacio Web, tres niveles de gestión:

\begin{tabular}{|l|l|}
\hline Directorio & $\begin{array}{c}21,565 \\
\text { revistas }\end{array}$ \\
\hline Catálogo & 6,742 \\
& revistas \\
\hline Enlace a Revistas Electrónicas & 5,085 \\
& revistas \\
\hline
\end{tabular}

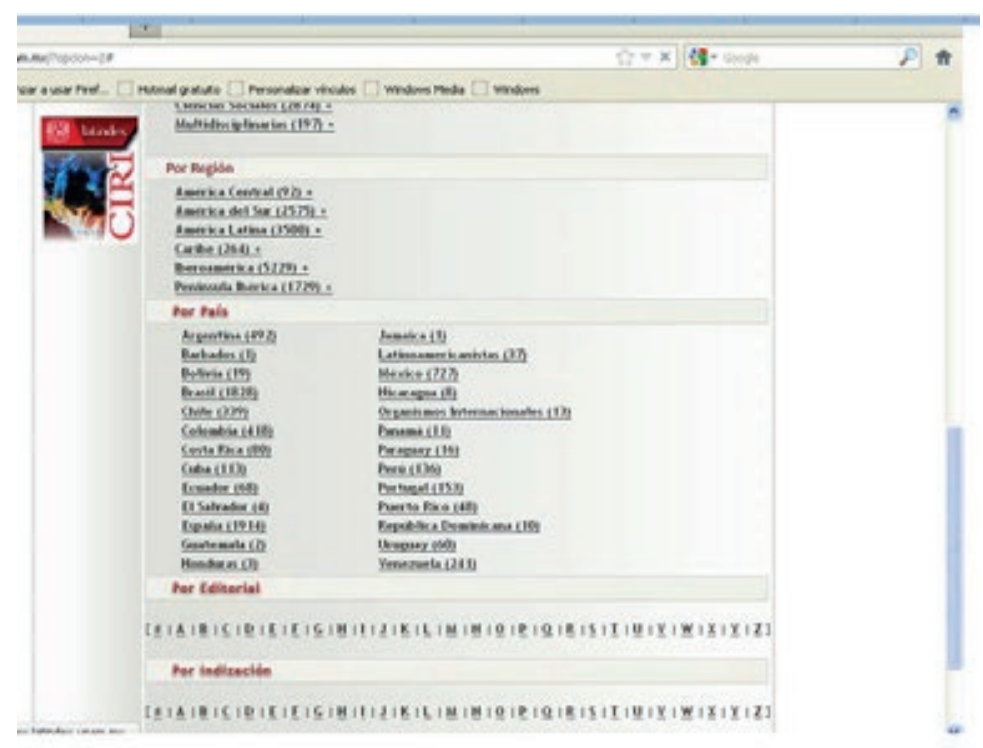

Figura 2. Cantidad de revistas en el sitio, por países en el espacio LATINDEX. Fuente - http://www.latindex.unam.mx/?opcion consultado el 24 de mayo 2013

El registro de Catálogo agrupa a 113 revistas cubanas científicas y especializadas, desde una perspectiva disciplinar no se registran revistas en Geografía, pero desde una concepción interdisciplinar la integran: Cuba: medioambiente y desarrollo; Ingeniería Hidráulica y Ambiental; Minería y Geología; Arquitectura y Urbanismo; Voluntad Hidráulica; Transporte, Desarrollo y Medioambiente entre otras que tratan contenidos de las ciencias geográficas desde sus carácter disciplinar, inter y transdisciplinar, y desde abordajes especializados y científicos. El sitio http://www.latindex.unam.mx/buscador/ficPais.html?opcion=2\&clave pais=12

brinda información detallada de las publicaciones adscritas a este sistema de acceso 
a contenidos. Demuestra el Portal que Cuba ocupa el 10mo. lugar en cantidad de revistas a difundir por este canal.(ver Figura 2.)

\subsection{Revista Cubana de Geografía}

La revista cubana de Geografía, del Instituto de Geografía Tropical (IGT) surge para cumplimentar un amplio propósito, responder a la demanda publicable de contenidos desarrollados a partir de investigaciones y proyectos en pos del desarrollo del país y de la colaboración internacional. Pretende de forma inmediata incorporarse a los estándares internacionales a través de varias acciones que en concreto respondan al plan estratégico del área de trabajo: desarrollo tecnológico y, el de la dirección del IGT, con vistas a potenciar la actividad científica, estas gestiones se puntualizan con:

- Participación en el Fórum del Instituto de Geografía Tropical para destacar sobre la importancia de las publicaciones científica

- Convocar a especialistas investigadores y a trabajadores en general a participar en cursos de Comunicación científica que se convocan en las instituciones cubanas

- Promover en otras organizaciones e instituciones, sobre el trabajo editorial de la revista y su convocatoria a publicar

Otras tareas están concebidas con vistas a registrar y acreditar a la revista en los sistemas nacionales e internacionales de difusión científica:

- Actualización de la Política Editorial

- Publicación de dos números en el 2013

- Comenzar el proceso de certificación CITMA para las revistas científicas, según resolución ministerial 59/2003

- Iniciar estrategias de posicionamiento hacia bases de datos y repositorios internacionales de difusión científica

- Realizar acciones investigativas para incorporar a la revista a la plataforma de gestión editorial Open Journal System (OJS), un conjunto de softwares identificados como códigos abiertos, los cuales asimilan y procesan, desde y para, parámetros internacionales de gestión y visibilidad científica.

\subsection{Valoración económica y aporte social}

La efectividad de la investigación y su valoración económica es un parámetro que incide en el impacto y las metas deseadas, es un tema que adolece aún de metodologías estándares para su medición, debido sobre todo a su complejidad, plantean investigadores en la temática (Gálvez González AM, 2003; 2010)

Se han realizado varios estudios sobre situación y perspectivas de las evaluaciones económicas en Cuba en aras de ganar en homogeneidad y transparencia de criterios, así como garantizar la posibilidad de reproducir los estudios que se presentan, los investigadores Gálvez González y otros (2004), exponen sobre métodos evaluativos, determinantes para la toma de decisiones e incentivar hacia las buenas prácticas en 
materia de evaluación económica, para sus planteamientos se apoyan en la situación del sector de Salud Pública en Cuba. Estas presentaciones formales de evaluaciones económicas por parte de autoridades institucionales responsables de la toma de decisiones son determinantes para la introducción de tecnologías de punta y otros objetivos de inversión y desarrollo.

Apuntan estudios que el crecimiento de las evaluaciones económicas en los últimos cinco años en Cuba, se ha producido a expensas de la producción científica en el ámbito docente e investigativo, donde el sector sanitario ha tenido una participación destacada. (Gálvez González, García Fariñas, Anaí; y otros 2012).

A partir de experiencias de los investigadores antes mencionados, se proponen un grupo de recomendaciones y tareas para estimular y fortalecer el uso de las valoraciones económica en los procesos de toma de decisiones y exponen como primer paso: Publicar resultados, guías y procedimientos metodológicos derivados de las investigaciones realizadas, (productos comunicativos de la actividad científica) de una forma viable y precisa, proceso que evidencia a la comunicación científica como un procedimiento inicial y determinante para las valoraciones económica en las instituciones del país y lograr así el impacto social proyectado. (Lozano Casanova; Saavedra Roche; Fernández Franch, 2011)

En Cuba la política de información y comunicación científica, es dictaminada por el CITMA la cual estipula y estimula la publicación y certificación de los contenidos científicos, estos se apoyan en su clasificación, las cuales se agrupan según las estipulaciones internacionales en:

Artículos científicos: contiene a una amplia tipología, que la integra a: los artículos de revisión, de investigación, de comunicaciones cortas, de reflexión, reseñas científicas, recensiones y otras modalidades que responden a las estrategias propias de publicaciones e instituciones.

Monografías y ensayos científicos: son documentos críticos, académicos y científicos, sus formas expresivas tienen en cuenta los contextos y objetivos hacia los públicos e igual toman sus particularidades de acuerdo a los desarrollos sociales que se procuren.

\section{CONCLUSIONES:}

Las investigaciones en las ciencias geográficas cubanas precisan de espacios validados en los entornos virtuales de Internet, la revista cubana de geografía está convocada y convoca a su comunidad a potenciar el proyecto. Publicar en la revista será un vehículo para resaltar el quehacer cubano.

La revista cubana de Geografía es un vehículo para la proyección socio-económica del conocimiento derivado de una constante búsqueda del saber, que se potencia de la capacidad y talento de nuestros investigadores, cualidades que se legitima por su aplicación y pertinencia, sus estrategias de trabajo están implementándose en 
función de una labor constante para su inserción en los estándares internacionales de posicionamiento.

Los procesos de valoración económica de la producción científica se conceptualizan dentro del impacto social, al ser estos participes de las políticas inversionistas en los diferentes sectores. Es importante implementar productos comunicativos de la actividad científica con características que lo distinga como preciso y viable, premisas que se traducen en trabajar en aras de una comunicación científica eficiente, rol que deben ocupar las revistas científicas.

Publicar es un proceso indispensable para todo creador, investigador o profesional, con esta acción se valida, comunica y aporta al conocimiento colectivo e individual en pos de beneficio e impacto social. Mantener una estrategia comunicativa en este sentido debe ser deber de todo especialista.

\section{REFERENCIAS}

\section{PUBLICACIONES PERIÓDICAS, REVISTAS, WEBES Y SIMILARES:}

Albornoz M,Estebanez ME,Alfaraz C.Alcances y limitaciones de la noción de impacto social de la ciencia y la tecnología. Revista CTS 2005;2(4):73-95. Disponible en

http://www.scielo.org.ar/pdf/cts/v2n4/v2n4a05.pdf

Araya Favia (2012) Educación Geográfica para la Sustentabilidad. Disponible en:

http://revistas. userena.cl/index.php/monographuls/article/view/18 Consultado el: 22de mayo 2013 Cita (Vancouver): Dorta Contreras AJ, Álvarez Díaz L. Producción científica de Cuba: una perspectiva desde la obra de dos mujeres académicas. Acimed 2007;16(5). Disponible en: http://bvs.sld.cu/revistas/aci/vol16 5 07/aci071107.htm [Consultado: día/mes/año].

Editorial Geotech. Documentos acreditativos, Departamento de Desarrollo Tecnológico, Instituto de Geografía Tropical, La Habana, Cuba. Consultados en mayo de 2013

Fernandez Polcuch. La medición del impacto social de la ciencia y la tecnología. OEI. 1999. Disponible en :http://www.oei.es/ctsiima/polcuch.pdf

Gálvez González AM. Guía metodológica para la evaluación económica en salud: Cuba, 2003. Rev Cubana Salud Pública [Internet]. 2004 [citado 30 de diciembre de 2011];30(1). Disponible en: http://scielo.sld.cu/scielo.php?script=sci arttext\&pid=S0864 $34662004000100005 \&$ lng=es\&nrm=iso\&tlng=es

García Fariñas A, Gálvez González AM, García Rodríguez JF. Aspectos metodológicos críticos en las evaluaciones económicas de salud en el contexto cubano. Rev Cubana Salud Pública [Internet]. 2010 [citado 30 de diciembre de 2011];36(3). Disponible en:

http://scielo.sld.cu/scielo.php?script=sci arttext\&pid=S0864-

34662010000300007\&lng=es\&nrm=iso\&tlng=es

Gálvez González AM, Álvarez Muñiz M, Sarduy Domínguez Y, Morales Laberón M. (2004). Diagnóstico de la evaluación económica en salud en Cuba. Rev Cubana Salud Pública

[Internet]. Citado 30 de diciembre de 2011;30(1). Disponible en:

http://scielo.sld.cu/scielo.php?script=sci arttext\&pid=S0864-

34662004000100003\&lng=es\&nrm=iso\&tlng=es 
Gálvez González,Ana María, Garcia Fariñas, Anaí; Portuondo Sánchez; Carmen, Bastanzuri, Cristina L. ; Manuel; Collazo Herrera (2012). Evaluación económica en salud y toma de decisiones en el contexto sanitario cubano. Revista Cubana Salud Pública vol.38 no.2 Ciudad de La Habana abr.-jun. 2012. Consultado el24 de mayo de 2013 http://scielo.sld.cu/scielo.php?pid=S0864 $34662012000200008 \&$ script=sci arttext

Libera Bonilla ES. Impacto, impacto social y evaluación del impacto. Acimed 2007; 15(3):2. Disponible en: http://bvs.sld.cu/revistas/aci/vol15 3 07/aci08307.htm

López Bastida J, Oliva J, Antoñanzas F, García Altés A, Gisbert R, Mar J, Puig J. Spanish recommendations on economic evaluation of health technologies. J Eur J Health Economic. 2010;11(5):513-20. Epub 2010 Apr 20.

\section{LIBROS, CAPÍTULOS DE LIBRO O ENTRADA DE UN LIBRO DE CONSULTA, INFORMES TÉCNICOS, TESIS}

Linares Herrara M. P. ; Santovenia Díaz J.R. (2012) Buenas Práctica: Comunicar e Informar, Editorial Academia; ministerio de Ciencia, Tecnología y Medioambiente, La Habana, Cuba

\section{Autores:}

Msc. Manuel Paulino Linares Herrera, graduado en la Universidad de La Habana, profesor en la facultad de Comunicación, especialista e investigador en la Academia de Ciencias de Cuba, La Habana, Editor de publicaciones Científicas. Doctorante en Ciencias de la Comunicación.

Lic. Ernesto Budiño Rodríguez, graduado en la Universidad de La Habana, labora como especialista en gestión web en el Instituto de Geografía Tropical, La Habana, Cuba

Msc. Orestes Sardiñas Gómez, graduado en la Universidad de La Habana, labora como especialista en investigaciones de las ciencias geogrñaficas en el Instituto de Geografía Tropical, La Habana, Cuba 